\title{
A NEW MODEL FOR SYNCHRONOUS MULTI AGENTS PRODUCTION AMONGST CLIENTS AND SUBCONTRACTORS
}

\author{
Mitrouchev, P.* \& Brun-Picard, D. ${ }^{* *}$ \\ *G-SCOP Laboratory, FRE-3028 CNRS, 46, av. Félix Viallet, 38031, GRENOBLE Cedex 1, France \\ ${ }^{* *}$ LSIS - UMR CNRS 6168, CER ENSAM - Equipe IMS, 2, cours des Arts et Métiers, \\ 13617 Aix-En-Provence Cedex 1, France \\ E-Mail: Peter.Mitrouchev@hmg.inpg.fr; Daniel.Brun-Picard@aix.ensam.fr
}

\begin{abstract}
In this paper a new Product Model (PM) for production is presented. An agent able to negotiate the operations that the product must undergo on the stations in manufacturing is attached to it. That agent has all the necessary information concerning its manufacturing range, the environment of production, the objectives, and the constraints and rules of production. The PM was introduced into a common experimental platform developed by the partners of the "Synchronous Production Amongst Clients and Subcontractors" (SPACS) Project. The latter integrates all the elements necessary for the management of the production in interaction with the Electronic Data Interchanges (EDI) amongst clients and subcontractors. A new approach for manufacturing systems' control, allowing increased reactivity and flexibility, is also proposed. It is based on a social approach with a totally decentralized architecture. The manufacturing system (MS) is made up of a set of autonomous and intelligent agents as in a society. The product is considered as an actor who takes part in the decisions. The products are likened to customers and the resources to service providers.

(Received in September 2006, accepted in March 2007. This paper was with the authors 2 months for 1 revision.)
\end{abstract}

Key Words: Product Model, Scheduling, Multi-agent System, Manufacturing Systems

\section{INTRODUCTION}

To remain competitive, companies must on the one hand offer ranges of increasingly diversified finished products and on the other hand renew their offer within an increasingly short timescale. Technological changes, in particular those concerning data processing, modify considerably the conditions of production. Forms of extended coordination of the activities are developing and the speed at which the nature of manufactured products evolves is increasing. Scheduling problems in practice were, for a long time, been the subject of many papers. Different tools and models are applied to resolve this problem like Petri nets, Simfactory II.5, Witness and Arena software [1, 2]. Let us remember that in static scheduling it is assumed that the number of jobs, their processing sequence and the respective processing time are known a priori. However, in real time situation there are many uncertainties associated with part arrival, processing time and machine availability. Some comparative studies of dispatching rules in the presence of varied rate of breakdown levels were presented in $[3,4]$. Mean tardiness, maximum tardiness and the number of tardy jobs objectives were used to evaluate the performance of each dispatching rule but minimisation of flow time based and tardiness based performance were not proposed. In most of studies it is assumed that machines in the shop are continuously available. However the risks as breakdown of machines for example are quite frequent in the Manufacturing Systems (MS).

The effect of some breakdown parameters on the dispatching rules was studied in [5]. Different values of breakdown parameter and utilization level were used in order to analyse 
their effect with respect to tardiness-based objectives. Today the performances of a MS depend on its scheduling performances. In this context, we consider that traditional scheduling approaches, using combinatory optimization, are inefficient because they fail to take into account the dynamic aspects, which are prevalent [6]. The disturbances (e.g. machine breakdowns, quality problems, organizational problems, modifications made by the commercial department, ...) lead to a difference between the real state of the shop floor and the prearranged schedule [4, 7]. The complex prearranged schedule has to be re-computed and the next solution may be very different. Hence, optimization must not deal with a hypothetical balance state but rather the dynamic state of the system. Thus, numerous methods perform the short term scheduling without prearranged schedule [8]. The scheduling is based on rules, which assign operations in real time in reaction to events on the shop floor. Those methods increase reactivity and allow a more efficient management of the complexity. However, it is impossible to anticipate the behaviour of the shop floor and quite difficult to balance the burden amongst the resources: unfavourable situations or blocking may appear.

Therefore, an enhanced organization amongst prediction, anticipation and reaction is required. Prediction concerns medium and long term scheduling, whereas anticipation concerns short term scheduling. Anticipation allows successive operations to be linked in time. The aim of this paper is to demonstrate that anticipation on a very short term allows a real time scheduling to be built based on the events of the shop floor. In some previous works $[6,9]$ we developed an approach called "Product approach". The latter is based on a social model in which the resources and the products are both active to schedule the operations. The suitable solution emerges from the negotiation of the operations between products and resources for a very short-term period.

In this context, we took part in Synchronous Production Amongst Clients and Subcontractors (SPACS) Project, subsidized by the Rhône-Alpes regional council. Its goal was to propose a global solution for the manufacturing control, aiming at the integration of the production system with the whole of the functions of the company. The starting points of our work are already existing developments of a multi-agents model of production ensuring short-term synchronization amongst clients and subcontractors [6, 10, 11]. Our work, within the SPACS Project, was more particularly related to i) the integration of the functions of design and manufacture, being articulated on a "Product Model" and ii) the piloting of the production by the product based on a model of society, the product being considered as an actor, as well as the stations of manufacturing. Those two aspects are the subject of this paper.

The rest of this paper is organized as follows. The objective of the research within the framework of SPACS Project is presented in section 2. Thereafter section 3 describes the simulation, reasoning, the results of the simulation and their validation. Section 4 describes the product as a control actor in a flexible manufacturing system. The following section 5 presents the insertion of the model in the SPACS platform. And finally discussions and conclusions are given in Section 6.

The results of this study may be useful to product and production-system designers enabling them: i) to establish a model, which is appropriate for the piloting of the production system, ii) to have all the information for the manufacturing of the product.

\section{OBJECTIVE OF RESEARCH WITHIN THE FRAMEWORK OF SPACS PROJECT}

The scientific goal of the project was to propose methods of synchronization which do not introduce an artificial constraint into the organization and the local management of the production. It consisted in:

- preserving as much as possible the autonomy of the partners, 
- proposing an architecture of decentralized decision,

- ensuring independence in order to effectively manage its resources, and to face against proper risks in its local system.

In such a decentralized context, it is necessary to have synchronization mechanisms based directly on the production targets, such as defined by the supply agreement to the customer.

The project also aimed at developing approaches and a methodological support adapted to the short-term scheduling of the production of Small and Middle Enterprises (SME) subcontractors, in good synchronization with the customer or partners companies. The impacts at the organisational, economical and operational levels of the installation of these approaches were also estimated [10].

\subsection{Consequences}

Consequently, relationships amongst client and subcontractor change. The credibility of a subcontractor does not only depend on the quality of the products and the strict respect of the deadlines. It also depends on its capacity of reaction to the inevitable dysfunctions occurring during the manufacturing. Thus we needed:

- a reliable support of communication in order to exchange information necessary to reactivates production management (cf. § 2.2),

- a dysfunctions' model in order to anticipate on their propagated effects (cf. § 4.5).

The margin of autonomy is essential to locally absorb the risks resulting from manufacturing processes or caused by the fluctuations of orders. The profitability of the companies able to answer the constraints of a synchronous production involves adequate contractual conditions which is a question to define.

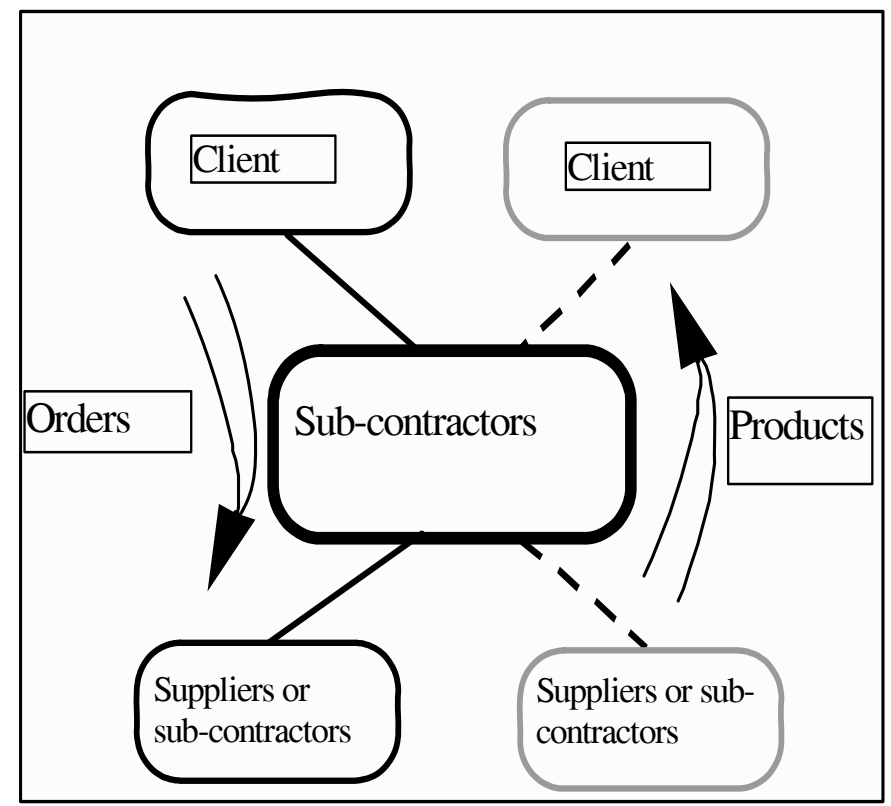

Figure 1: General representation of the relations between clients and subcontractors.

Thus, the production system becomes reactive, characterized by a synchronous approach, rather than interactive, characterized by an asynchronous approach. The project aimed at developing approaches and a methodological support adapted to the short-term scheduling of the production of sub-contracting SME, in good synchronization with the customers or partner companies. We also estimated the impacts at the organisational, economical and operational level of the installation of these approaches. 


\subsection{State of Industrial art. Supply agreements}

Let us recall the traditional approaches of prescriptive type [12] where: i) the products are inert; ii) the operators are slaves; iii) all depends on the person in charge of the planning. The open (estimated) and the firm orders were generated via Electronic Data Interchanges (EDI) [8]. The EDI is a teleprocessing application allowing, according to a standardized format, the exchange of computerized documents amongst the computers of partners (commercial or others). Then it allows the integration of these documents, once received, validated and accepted, into the file of work of the computer of the recipient and their possible immediate processing. That allowed us:

- to split the quantities to be delivered (a delivery per day for example),

- to make firm these quantities only at the last moment (a few days, even a few hours).

Among the adopted solutions we note:

- using better the open (estimated) orders and EDI in order to reduce the wastes of time and the margins of error,

- producing with in just by integrating the client delivery orders into the production schedule of subcontractor (a margin may be required),

- reducing this margin, without risk of rupture of provisioning for the customer, it was proposed to soften the supply agreement,

- in certain cases giving the subcontractors the possibility not to provide the requested quantities, in the express condition that they warn the customer early enough.

We proposed to open the supply agreement towards a subcontractor or groups of subcontractors who are not subjected to the same risks at the same time.

\subsection{Problematic and reasoning}

The suggested model is based on the running of the production by the product and on a model of society [6]. As we said the product is considered as an actor. Whereas for the traditional approaches of prescriptive type the products are inert, the operators are slaves and all goes up with the responsibility for planning, our approach is based on a model of society in which:

- the products choose their operators, (autonomy of the actors),

- the products and the operators make an appointment, (production guided by the product),

All the actors of this society collaborate with the same aim, to satisfy objectives laid down by the production plan. The decisions are made in co-ordination with all the concerned actors. Each one acts at the same time at the estimated level in short run (appointment) and at the reactive level (reassignment of the appointments in the case of disturbances). That supposes coherence and a continuity of the whole activities of Design-Management-Production in close relation with the Integrated Design, without forgetting the determining role of the human being in the capacity as intelligent actor, factor of autonomy.

The implemented mechanism is based on rules of good direction used in client/subcontractor relations with service providers working on return. These rules are distributed in an identical way on each actor and are applied in parallel and in real time.

\section{SIMULATION, REASONING, RESULTS AND VALIDATION}

Among the industrial partners associated to the project, the companies of the undercutting sector played a particular role, because they were intended to be used as models for the construction of a platform on which simulations would be carried out. Thus the model presented in this study was tested on a platform representing a company of undercutting, supplier for the car industry Renault Industrial Vehicles (RIV). 
The collection of necessary data required the presence for a long time of researchers on the factory's site. The first analysis carried out in situ allowed collecting a precise description of matter flows among the various activities of parts production. It also allowed a careful reflection on the significance of the synchronous production and its implications in term of production management. Then, the analysis could be widened by means of the regular participation of a researcher in the installation of new software of production control PRODSTAR $[10,12,13]$ on the site of a partner company.

The software allowed the commercial management, the management of the purchases, the management of technical data, quality and production control to be taken into account. Initially the level of production management was limited to a level of planning of "reactive oriented product method" (ROPM) type. Thus a data base of the technological platform was constituted to which the model presented above was applied. The validation of the suggested concepts was realized through the simulation presented below.

\subsection{Evolution. Safety and flows simulation}

After a planning optimization we proposed an evaluation of the safety of flows compared to the capacities of the subcontractors according to three stages:

- model for total planning,

- simulation of flows and multicriterion assistance for results’ interpretation,

- data-processing support.

Flows simulation presupposes that random dysfunctions and decisions are taken according to criteria which measure simultaneously safety and delays compared to the planning. The goal was to provide subcontractors with rules of scheduling which give satisfactory performances in term of safety and productivity. Interaction modelling with the partners by stochastic processes was adopted. It is the case of:

- the arrival of EDI from the client,

- the delivery of subcontractors,

- the delivery of possible suppliers.

Simulation consisted in replacing a system by a logico-mathematical model. Then this model was translated into a program able to generate data characteristic of the system. And finally that data (average value, confidence intervals...) was interpreted statistically in order to draw some inferences from it. The adopted approach for simulation was the approach by process. Various software were tested (WITNESS, Simfactory, ARENA, ...) in order to retain the one which would be the most adapted to represent the problem in accessible form for the subcontractors. The software was also supposed to have a sufficient data-processing opening in order to articulate a level of managing, required by each of our various approaches of synchronization. This choice seemed very important to us because beyond the validation of the concepts, subcontractors could use the simulation as a decision-making aid. Finally the ARENA/SIMAN software was retained allowing:

- the system's structure description: the processes are described by predefined functional blocks (size of a resource, transport of a part, machines, transport systems, storage...),

- the description of entities which represent orders, parts or information: the algorithm of change of state is known in a block. The block can call upon a bookshop of preset procedures or upon a procedure user which describes the decision rules,

- the definition of the running rules which make it possible to direct the entities in the system: the activities are launched by the passage of entities in the functional blocks, the system's state is characterized by aggregate variables and attributes carried by the entities.

This software, having a graphic animation, also allows the visualization of the evolution of the system's state (circulation of the parts in the workshops, activities of the machines, 
composition of stocks). At any moment of the simulation it was possible to obtain information on the state of an object (machine, entity...) and to carry out statistical analyses. The production process of the Pernat Company partner was studied as an example. It is summarized by Fig. 2.

\subsection{Multicriterion interpretation of the results}

Let us remember that the simulation of a system with discrete events provides performances' measurements of the system. In this study of a MS (Pernat subcontractor enterprise) we were more particularly interested in the following performances:

- position of the delivery date compared to the interval planning,

- safety of the production process.

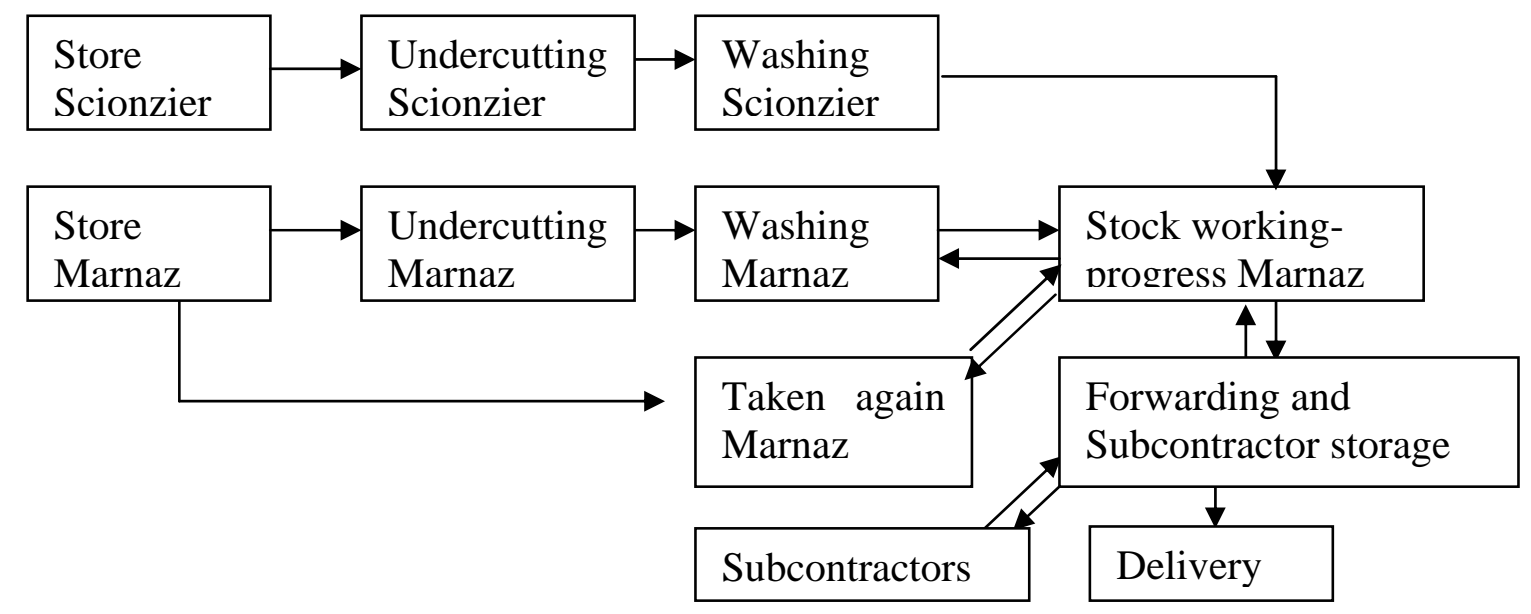

Figure 2: Flows' simulation and evolution.

The simulation provided a confidence interval for each one of these performances. To compare two control strategies one is often confronted with a problem of multicriterion decision. However, a decision generally implies several criteria, which can be qualitative or quantitative. This is a complex process which required the implementation of a methodology. The object of the decision was initially delimited, and then all the alternatives were given. After then the criteria were determined and a part of them aggregated. Lastly, a procedure which allows the best possible solutions to be chosen according to considered criteria was sought. Let us note that to determine the final solution, a human intervention is sometimes necessary.

Quantitative criteria were chosen in this study. Such a criterion is a function of set of alternatives towards a completely ordered set. The criterion of performance was the position compared to the interval generated by planning. The criterion relating to safety was then defined. The procedure of selection thereafter was sought and adopted. Let us recall that there exist three main approaches for procedure selection:

- aggregation in a single criterion,

- outclassing (on classification),

- interactive interactions.

Among these possibilities the third one was adopted.

Thereafter, we took interest in the definition of the "measurement" of performance related to safety of flows between the clients and sub-contractors. This safety is the criterion which was taken into account in the process of decision-making aid, in addition of the position of the delivery dates compared to the intervals of planning. This criterion was finally included in the simulation model. 


\subsection{Information exchange and activities' synchronization}

The Laboratory of Software for Computer-Integrated Manufacturing (LSCIM) developed an interface organizing the exchanges of information and synchronizations of activities called PROPILOT [14]. Firstly the simulation made it possible to reproduce the operation of the undercutting company, then to apply the models of piloting in order to estimate their effectiveness. This analysis required to define several criteria of quantitative and qualitative evaluation (delay of a part, level of stock, cost, ...). It was thereafter possible to analyze the received requests by the subcontractor in order to evaluate its limits and over costs generated by the rush orders and by a lack of anticipation on the client's part.

\section{THE PRODUCT AS A CONTROL ACTOR IN A FLEXIBLE MANUFACTURING SYSTEM (FMS)}

Let us remember that in the classical approach the problem of manufacturing control is normally approached by the means of the planning and the scheduling. The problem is posed in terms of production flow and of resource allocation. The outcome of these steps is classically Gantt chart that freezes the operations allocated to each resource, in function of time. This approach can be said to be based on manufacturing operations and is characterized by the answer to three questions which are, in order: "Who does the operation? - When does the operation take place? - What or which product is concerned by the operation? [14].

The product approach, proposed here, aims at higher flexibility and reactivity. It is based on a society model where the members are products and resources. This approach leads to rearranging the order of the questions: What? Who? When? The product becomes an active element of the production system and takes part in the decisions which define its control. Each product communicates and negotiates with all the resources to make appointments for each operation. The products are like customers and the resources are service providers: thus, the production system is made up of a set of autonomous and intelligent agents able to cooperate to achieve the global goal of the system.

The fundamental principle of product approach leads to associate all the knowledge and all the decision capacity required for the control to the current products. Thus, the product possesses the specific knowledge to search for and treat the information on the production process: production goals, decision rules, equipment features and production environment [9]. This specific knowledge base contains all information on the product including:

- its identity, its functional and structural features, its parameters,

- the process and the operational sequence to produce the product,

- the priority weights,

- the equipment features and production environment.

It also contains the information on the prearranged schedule, the real state of advancement and of quality of its operations, production goals and decision rules. This information or knowledge is used to find out a heuristic solution to achieve the planned objectives, taking into account the unexpected events. Resources are also autonomous and have a specific knowledge base containing the required information to perform operations.

Two special entities are introduced to complete this structure.

- The first entity specializes in supervision and man/machine relationships. It acts when human decision is essential. Thanks to this entity, overall safety of the system is increased because the human operator is always informed and associated with the quantitative decisions.

- The second special entity is an expert system which gives to each product all the initial information and the specific knowledge base required to schedule and control the system. 
The behaviour of these two entities depends on the global environment, the global production goals and events occurring. They form the link between the planning level which defines the manufacturing objectives and the control of the system [9]. Their global knowledge insures global consistency and vertical integration of production data. To establish a link with the CODECO approach (COordinated DEcentralized COntrol) [7], these specialized entities represent the coordination level. However, they do not interfere with the decision process and each execution entity is totally autonomous. The function of the specialized entity is to prepare the production context and the decision framework to allow harmonized behaviour of the global MS.

\subsection{Architecture of the product-oriented approach}

The proposed product approach is characterized by the decentralization of the control. The control is based on a set of autonomous, homogenous and cooperative entities. In some previous researches, we developed a totally decentralized control approach $[9,15]$ in which each "execution entity" controls an element (or resource) of the operative system. These execution entities are completed by specialized entities for man/machine relationship and supervision. The new step in this approach is to consider that products are execution entities (Fig. 3). Thus, products become actors of the MS.

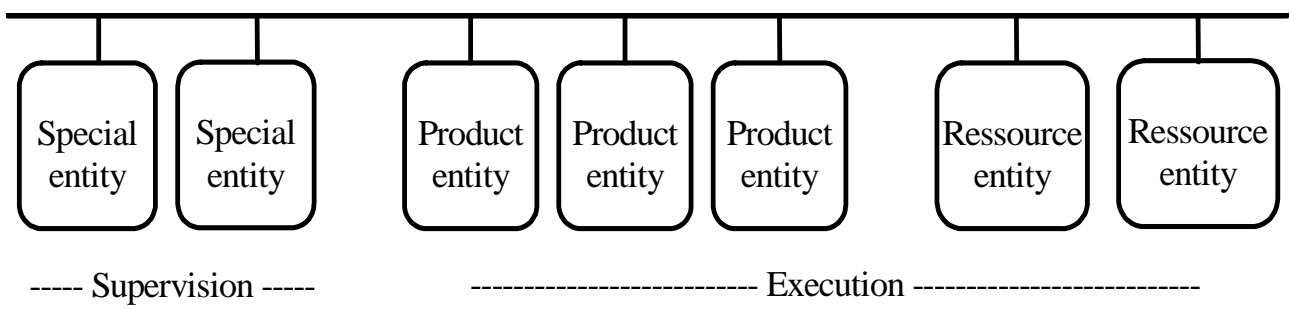

Figure 3: Organization of the decentralized control.

The fundamental principle of control decentralization relies on this homogeneous set of elements taking and executing decisions at the same time. These elements are autonomous, cooperative and coordinated by their goals. Each entity has the ability of "Communication", "Decision" and "Action" and possesses a local database.

The Action represents the ability to control the physical part of the process. An action corresponds to an elementary operation of the process.

The Decision gives the ability to coordinate the actions of an entity with the other entities and to face unexpected events.

The Communication creates the link for cooperation and information exchange between entities.

This decentralized approach simplifies the control of the MS. It attaches a particular importance to events which involve actions [15]. The autonomy gives to each module the ability to locally eliminate disturbances and, as a consequence, the system reacts as close as possible to the place where events occur [7]. Furthermore, with this approach, the architecture of the control system allows a complete integration of all the control levels, into a homogeneous, modular and open structure.

\subsection{Self-scheduling and control driven by the product}

As we said, each product is considered as a service caller or a client. The product is able to communicate and to negotiate with the other autonomous entities, resources or servers, in order to find out the best schedule. In this way, the products are able not only to organize and 
control the system but also to control the machines. Each product keeps an agenda in which it records the operations to carry out. For each operation the identity of the server, the beginning and end dates are recorded. The resources are also autonomous and are able to accept or to refuse the client's requests. They also have an agenda. In order to avoid the combinatory explosion and to reduce the computing time, the product searches to the best end of the operation time for two or three consecutive operations. In the disruption case, the autonomous entities react rapidly and locally. To this end, the appointments with the stopped machine are cancelled and the products try to find another machine.

The product takes the initiative for negotiating the appointment because it has the information on its operation sequence and their approximate duration, its due date and its operation progress. It also knows the suitable resources for each operation. The negotiation protocol is the following:

1. For the first operation to carry out, the product communicates with all the suitable resources and makes a provisional appointment. A beginning date and an end date are negotiated. The product takes into account the transportation time and the resource takes into account its potential setup-time.

2. For the next operation, if there are still operations for the product, it communicates with all the suitable resources and makes provisional appointments. Thus, the product obtains one or more appointment sequences for two consecutive operations. It could communicate and make provisional appointments for the next remaining operations.

3. Then, the product chooses the best sequence of operations and communicates with all the machines to confirm the chosen appointments and to cancel the others.

If a breakdown occurs, the autonomous entities react locally and rapidly: the appointments with a stopped machine are cancelled and the product tries to find another machine [8].

If a conflict for an appointment request occurs, the product with the highest priority makes its appointment before the other. Many usual decision rules may be used such as: FIFO (First In, First Out), LIFO (Last In, First Out), SPT (Shortest Processing Time), EDD (Earliest Due Date), MOR (Most Operation Remaining), FOR (Fewest Operation Remaining), etc.

\subsection{Quality of solutions and functions of supervision}

Each product and each resource aim at satisfying their own criteria. The quality of the global solution (in terms of productivity) depends on the proper succession of all the operations for all the products on all the resources of the manufacturing system. Products and resources do not take into account the global state of the system and only solve local problems. However, the presented negotiation protocol allows consecutive operations to be linked to find favourable sequences, to limit the waiting time for the products, the idle time for the resources, the transport time and the setup-time.

A near just-in-time behaviour with a steady flow of the production is expected, though it cannot be absolutely demonstrated (in the state of our work and other known work). Effectively, with the appointment mechanism and the use of priority rules, each product is programmed to progress as fast as possible and each resource is to sequence operations with a minimum of lost time.

\subsection{Simulation}

This approach has been validated with the SMECI software. SMECI is a program environment for development of knowledge-based systems in various fields such as design, simulation, complex diagnostics and planning. In SMECI environment, all entities (products or machines) have been represented in form of objects that possess the necessary information for communication, negotiation and decision. Using this interface, it is possible to implement 
our approach and to have real time simulation of a Flexible Manufacturing System (FMS) functioning [8]. We modelled the prototype of a FMS, which is shown in figure 4 below. This system is made up of:

- an automated storage and retrieval system (AS/RS),

- a storekeeper robot (R1),

- three process robots (R2-R4),

- four belt conveyors (a conveyor for each robot),

- a central conveyor.

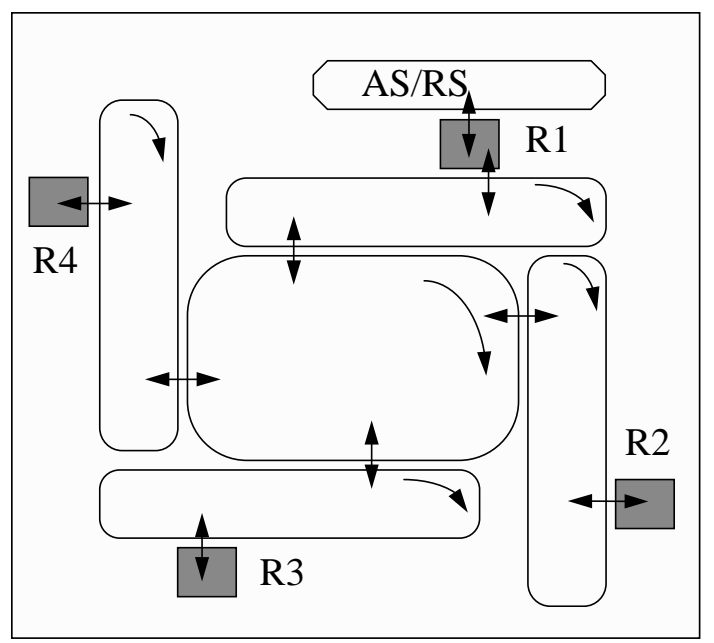

Figure 4: Structure of the FMS.

We assume that:

- the robots are able to carry out six different operations with a specific tool, a different setup time and a different processing time,

- the operation process is made up of no more than six different operations, but one operation can be repeated several times,

- the priority weight of each product depends on its due-date and on the estimated duration of the remaining operations, which can be increased depending on the latest end of operation times.

In the normal case, the priority weight of all products can be equal, but if a product is late, it can overtake the other by increasing its priority weight.

\subsection{Conflict problem and coordination mechanism}

To avoid communication conflicts amongst products and machines, only one communication token is used for all the products that are present on the conveyor. In this way, at any one time, only one product can communicate with machines and request appointments. This is not a problem because the required time to make an appointment is very short. If two or more products need the token simultaneously, the product with the smallest ratio of available time over the priority weight will obtain it.

The coordination mechanism resembles the appointment mechanism described in $\S 4.1$. When a product tries to find a machine for assigning one of its operations, it communicates with all the suitable machines. The products have all the required information to compute the transport time from one machine to another. The resources have all the required information to find out the setup time and the duration of operations. Furthermore each resource and each product has an agenda (appointment book). These agendas represent the negotiated appointments amongst products and resources, the operations to carry out, the beginning and 
ending times ... The interactive procedure between a product and resources is organized in four steps.

The product requests the earliest available date for each machine and the expected duration for this operation. It checks if it is possible to reach the machine before this date and propose a provisional appointment.

Considering the first step results, if the product has outstanding operations, the product communicates again to request appointment for the next operation. The product keeps the solution, which gives the best end time for the second operation (or for the first operation if it is the last). It communicates once again to confirm the chosen appointments for the 2 consecutive operations. If the product has a high level of priority, it immediately makes another sequence of appointments.

Afterwards, the product goes to the appointments to carry out its operations. The appointment mechanism begins again when its last operation ends, in order to take into account the effective end time of this operation.

\subsection{Disruption case. Simulation results}

When a breakdown is detected for a machine, its appointments that have not been carried out are not valid and the products that were assigned to this machine have to find another one. We choose to cancel all the next appointments with all the machines and to restart the appointment procedure. This proposal is motivated by two main raisons:

- each product keeps a position corresponding to its priority rank,

- the number of products on the conveyor is limited and the communication times are very short compared with the operation time. When the failure is set all the appointments are cancelled and the appointment procedure begins again.

In the first simulation, we assumed that:

- there is no breakdown in the production system, and the priority weight is the same for each product,

- the machines are able to carry out several operations and an operation can be carried out on several machines,

- the conveyor capacity is unlimited.

The obtained results show that, for this system:

- On average:

0 the machine utilization rate is about $87 \%$,

0 for the products the operating time rate is about $49 \%$ and the waiting time rate is about $43 \%$.

- A decrease in the number of products present on the conveyor entails a decrease of the waiting time rate.

- A reduction in the number of operations that each machine can carry out entails an increase in the waiting time and a decrease in the transport times.

- The increase in the priority weight does not change the global results.

Generally, in this first situation, the machine's utilization rate is always more than $86 \%$. In the second situation, breakdown constraints were imposed. On average, the machine utilization rate, the transport time and the waiting time increase. In general a very good rate of occupation for the machines (almost identical to the first case) has been obtained but the rate of the transport time has increased by $30 \%$. The best conditions for self-scheduling are observed in the case where duration of the operations is not very varied. Thus, it is interesting, if possible, to group the small operations in one operation and to subdivide the long operations. 


\section{INSERTION IN THE SPACS PLATFORM}

The Product Model presented in this study was tested on a platform of simulation, developed within the framework of the SPACS project. The tests were carried out starting from an industrial data file, received from Pernat Company, supplier for RIV. The Laboratory of Software for Computer-Integrated Manufacturing (LSCIM) developed an interface organizing the exchanges of information and synchronizations of activities called PROPILOT [14]. The other software necessary to the integration of manufacturing (data exchange, management of the technical data, management and administration of the sales, calculation of the needs, planning, launching, control and followed production) was chosen by all the partners of the SPACS Project, among standard software available on the market (WITNESS, SMECI, ...).

The Product Model with its descriptive part is at the heart of the management of the technical data and brings a clear structuring of the product data, accessible at the same time to the designer and to the manufacturer of the product. By its active part (produced agent), it forms part of the functions of control and follow-up of production. Thus, the Product Model brings a complete continuity of information, from the product design to the delivery.

\section{CONCLUSION}

Firstly in this paper a Product Model (PM) for manufacture has been presented. It was implanted in a common experimental platform developed by all the partners of SPACS Project. The PM integrates all the necessary elements to the piloting of the production in interaction with the EDI (Electronic Data Interchanges) amongst clients and subcontractors. The platform is also essential to direct the steps of research towards the difficulties encountered by the machine operators. It is also the support of demonstration and validation of the methods and tools under development.

Secondly we have presented the product-oriented approach, which considers three objectives simultaneously: flexibility, reactivity and modularity. To this end, a self-scheduling approach and control approach in which the products as clients are autonomous and intelligent entities has been presented. Each product has all the information on its manufacturing process (operation sequence, production rules, priority rule, due date, ...) and has a direct access to the information of all the other entities. The results are very encouraging when this approach is applied, because the machine utilization rate in two cases (normal case and disruption case) is high. Moreover, using this approach the waiting time is decreased and a quasi just-in-time control is obtained. The results also show that our approach is able to schedule and control the production system without any prearranged schedule.

However, proper operation requires a good balance between the load and the capacity of the workshop. This is obtained by spreading the fabrication orders to avoid waves in the workshop and jams at the entrance. Therefore, thanks to the autonomy of product entities and resource entities, our model allows an immediate reactivity of the system. Meanwhile, it aims at an efficient use of the resources thanks to the use of special entities specialized in supervision and in human-machine relationships.

Outlines:

With a view to a synchronous production amongst clients and subcontractors the effort in this study was related to the concept of process. Thus in the near future, we can hope to be able to solve the problems of synchronization and optimization of process. A mathematical model can be considered which will make it possible to locate the managing points of production system into which relevant information will be injected. We think that this model will bring a rigorous base to carry out possible improvements in the management of coherence and to possibly dysfunctions diagnoses. 


\section{ACKNOWLEDGEMENT}

The work described in this paper is a part of the SPACS research project that was supported by a grant from the Rhône-Alpes regional council.

\section{REFERENCES}

[1] Buchmeister, B.; Polajnar, A.; Pandza, K. (2002). Simulation study of effects of resources' downtimes on shop performance, International Journal of Simulation Modelling, Vol. 1, No. 1, 23-30

[2] Jain, A.; Jain, P. K.; Singh, I. P. (2006). Performance modelling of FMS with flexible process plans - A Petri net approach, International Journal of Simulation Modelling, Vol. 5, No. 3, 101 113, doi:10.2507/IJSIMM05(3)2.064

[3] Jain, A.; Jain, P. K.; Singh, I. P. (2004). An investigation on the performance of dispatching rules in FMS scheduling, International Journal of Simulation Modelling, Vol. 3, No. 2-3, 49-60

[4] Singh, A.; Mehta, N. K.; Jain, P. K. (2005). Tardiness based new dispatching rules for shop scheduling with unreliable machines, International Journal of Simulation Modelling, Vol. 4, No. 1, 5-16, doi:10.2507/IJSIMM04(1)1.036

[5] Holthaus, O. (1999). Scheduling in job-shop with machine breakdown: an experimental study, Computers and Industrial Engineering, Vol. 36, 137-162

[6] Mitrouchev, P.; Brun-Picard, D. (2005). A model for multi-agent distributed production system, Proceedings of Integrated Design and Production, CPI'2005, November 9-11, 2005, Casablanca, Morocco, CD-ROM, article No. 52, thème 2, 23 pages

[7] Baillet, P. (1994). Contribution à l'amélioration de la réactivité des systèmes de gestion de production par la mise en œuvre du concept de décentralisation des fonctions de décision, Thèse de doctorat, Université d'Aix-Marseille III, Marseille

[8] Manier, H. (1995). Contribution au pilotage d'ateliers flexibles réactifs, Thèse de doctorat, Université de Franche Comté, Besançon

[9] Brun-Picard, D.; Baboli, H. A. (1995). Self-scheduling for Flexible Manufacturing Systems: a product oriented approach, Proceedings of the 13th International Conference on Production Research (E. M. Dar-El, R. Karni, Y. T. Herer Eds.), Freund Publishing Company Ltd., London, England, 306-308

[10] Brun-Picard, D.; Mitrouchev, P. (1997). Production Synchrone Entre Donneurs d'Ordre et Soustraitants, Rapport d'activité pour l'année 3, mars 1997, Laboratoire 3S, Grenoble

[11] Mitrouchev, P.; Brun-Picard, D.; Hollard, M.; Haurat, A. (1998). A New Product-Model for Production, Proceeding of the 2nd International Conference on Integrated Design and Manufacturing in Mechanical Engineering, I.D.M.M.E.'98, May 27-29, Compiègne, ISBN: 2913087-03-5, Vol. 4, 1179-1186

[12] Brun-Picard, D.; Bouvet, H.; Baboli, H.; Binder, Z. (1997). The product as an active element of distributed production control, Conference on control of industrial systems, CIS'97, IFAC-IFIPIMACS, Belfort, Vol. 3, 359-365

[13] Bouvet, H. (1996). L'entreprise Pernat : Compte rendu de visites. Exposé PSEDOS, Grenoble 06.02.1996

[14] Dindeleux, R.; Berrah, L.; Haurat, A. (1998). A Formal Modelling of Control Processes, European Journal of Operational Research, Vol. 109, No. 2, 377-389

[15] Ferrarini, A.; Couvreur, L.; Brun-Picard, D. (1993). A new decentralized approach for F.M.S. control, Computer in design manufacturing and production, COMPEURO'93, IEEE Computer Society press, Los Alamitos, California, 410-416 